\title{
Relationship between MRI, Arthroscopic and Clinical Findings in TMD: Our Experience in Manitoba
}

\author{
Reda Elgazzar ${ }^{1^{*}}$ and Zhendan Pan ${ }^{2}$ \\ ${ }^{1}$ Oral and Maxillofacial Surgeon, Dr. Niznick College of Dentistry, University of Manitoba and WRHA Hospitals, Winnipeg, Canada
}

${ }^{2}$ General Dental Practitioner, Winnipeg, MB, Canada

"Corresponding author: Reda Elgazzar, Oral and Maxillofacial Surgery, Dental Diagnostic and Surgical Sciences, Bannatyne Ave, Winnipeg, Manitoba, Canada, Tel: +1(204)789-3634; Fax: +1(204)272-3077; E-mail: reda.elgazzar@umanitoba.ca

Received date: August 16, 2018; Accepted date: September 11, 2018; Published date: September 13, 2018

Copyright: (c) 2018 Elgazzar R, et al. This is an open-access article distributed under the terms of the Creative Commons Attribution License, which permits unrestricted use, distribution, and reproduction in any medium, provided the original author and source are credited.

\begin{abstract}
Objectives: To study correlations between magnetic resonance imaging, arthroscopic and clinical findings in patients with temporomandibular joint disorders in Manitoba.

Study Patients and Methods: A retrospective chart review was conducted on temporomandibular joint disorders patients who were diagnosed with MRI scan and treated with arthroscopic lysis and lavage at Health Sciences Centre and Seven Oaks General Hospital from 2006-2012. A data capture sheet collected clinical findings, disc position inferred from MRI scan and arthroscopic findings from patient records. The data was analyzed using the Minitab 15 Statistical Package.

Results: Eighty-seven joints of 58 patients were evaluated with the average age of 36.9 years and mean follow up of 6 months. MRI showed $52 \%$ of joints with anterior disc displacement without reduction. Arthroscopic findings showed adhesions (88.5\%), hyperemia (47.1\%), and synovitis $(31.0 \%)$ regardless of stages of internal derangement. There was a significant improvement in pain $(P<0.05)$, and in the Interincisal Distance (IID) (from 29.2 $\mathrm{mm}$ to $34.4 \mathrm{~mm}$ ) postoperatively.
\end{abstract}

Conclusion: Although arthroscopic findings did not correlate with disc position as per MRI scan, arthroscopic lysis and lavage has significantly improved pain, and jaw range of motion.

Keywords: TMJ arthroscopy; MRI; TMD; Internal derangement

\section{Introduction}

Temporomandibular Disorder (TMD) is an encompassing term used to describe any characterizations of pain and/or dysfunction of the Temporomandibular Joint (TMJ) and associated structures. Due to the broad range of physiopathological, psychosocial and traumatic factors which can be manifested as TMD [1] the first step in effective treatment is an accurate diagnosis.

Magnetic Resonance Imaging (MRI) analysis of the TMJ is currently part of the standard of care in the diagnosis of TMD [2]. Relationships between MRI findings and patient signs and symptoms have clinically been recognized [2-4] and the anatomic information provided can allow clinicians to further diagnose TMD. For patients who failed to respond to conservative measures for TMJ related pain with internal derangement, arthroscopic lysis and lavage have been shown to be an effective treatment modality to reduce pain, improve joint movement and range of motion [3-5]. While previous studies have shown success rates of $78 \%$ and higher, [3-5] the treatment is not guaranteed in effectiveness and there are no recognized standards for evaluating arthroscopic findings and for predicting subsequent postoperative outcomes from the preoperative assessment. It is controversial whether or not the changes in arthroscopic findings parallel the prognosis of symptoms. Some studies [6] showed that clinical signs and symptoms of TMD can be alleviated by non-surgical and arthroscopic treatments but most discs still remained anteriorly displaced without reduction despite treatment. It has also been found that the identification of disc displacement alone is not sufficient for surgical intervention as asymptomatic disc displacement of the TMJ has been observed in healthy volunteers as well. This suggests the position of the disc may not correlate with patient problems.

Although MRI is a great diagnostic tool for TMJ derangement, it is questionable whether it is necessary before proceeding to therapeutic arthroscopy and whether it correlates with arthroscopic findings and patient signs and symptoms. The purpose of this study was to examine the correlation between MRI findings, arthroscopic findings and clinical signs and symptoms pre and post arthroscopy in patients with TMJ internal derangement.

\section{Patients and Methods}

A retrospective cohort study was conducted to address the research goals. During the study period between 2006 and 2012, a total of 129 TMD cases from 84 patients were diagnosed by clinical examination and MRI and treated with arthroscopy lysis and lavage at Health Sciences Centre (HSC) and Seven Oaks General Hospital in Winnipeg, Manitoba, Canada. Exclusion criteria were set to remove patients from the study who were missing information on clinical findings, MRI reports and arthroscopic findings. As a result, 27 patients were excluded from this study. Our study group consisted of 87 TMJ in 58 patients comprised of 7 males and 51 females with a mean age of 34.5 
Page 2 of 4

years old and an age range of 16 to 74 at the time of surgery. Follow up times ranged from 1 month to 2 years with a mean of 6 months.

All patients had previously undergone unsuccessful nonsurgical treatment and had a variety of internal derangement grades according to the Wilkes Classification [7]. Before arthroscopic treatment, patients were examined clinically as well as with MRI. Clinical examinations consisted of inspections of the TMJ area and measurements of mandibular movements including maximum mouth opening, excursions and protrusions. All MRIs were performed at HSC and the images were interpreted by radiologists at HSC and reviewed by an attending oral maxillofacial surgeon. Arthroscopic lysis and lavage was performed using 2.4/ 30 degree scope (Stryker). Following arthroscopy, patients continued to follow conservative measures of treatment including analgesia (NSAIDs), muscle relaxants and physiotherapy for another 2 to 3 weeks.

A data capture sheet was created to record clinical findings pre and post arthroscopy, MRI reports of the disc positions including anterior disc displacement with reduction (ADDR) or anterior disc displacement without reduction (ADD), arthroscopic findings and conditions of the joint as well as medication used. A database was constructed and the data was anonymized with each clinical case given a unique ID to represent the patient to protect confidentiality and avoid bias. Minitab 15 Statistical Software Package was utilized for all data analysis. Probabilities of $<0.05$ were considered to be significant.

Ethical approval was granted by the University of Manitoba Research Ethics Board and further approval was obtained through the Winnipeg Regional Health Authority for Health Science Centre and Seven Oaks General Hospital as well.

\section{Results}

Of the 58 evaluated patients in our study, $12(13.8 \%)$ were male and 75 (86.2\%) were female with the mean age of 36.9 years (range, 16-74 years). Unilateral TMJ involvement was present in 35 (40.2\%) cases whereas bilateral TMJ involvement was present in 50 (57.5\%) cases. There were $2(2.3 \%)$ cases in which the involvement of the joint side was not specified.

\section{Pre- and postoperative clinical findings}

Pre- and postoperative clinical findings are as reported in Table 1. Pain, locking and clicking were the most common symptoms in patients undergoing arthroscopic treatment. At the postoperative follow-up, all signs and symptoms showed improvement, particularly cases with pain (42.5\%), locking (9.2\%), and clicking (18.4\%).

\begin{tabular}{|c|c|c|c|c|c|}
\hline & \multicolumn{2}{|l|}{ Preoperative } & \multicolumn{2}{|l|}{ Postoperative } & \multirow{3}{*}{$\begin{array}{l}\mathbf{P} \\
<0.05\end{array}$} \\
\hline & No. of joints & $\%$ & No. of joints & $\%$ & \\
\hline Pain & 81 & 93.1 & 37 & 42.5 & \\
\hline Clicking & 40 & 46 & 16 & 18.4 & $>0.05$ \\
\hline Crepitation & 16 & 18.4 & 6 & 6.9 & $>0.05$ \\
\hline Locking & 41 & 47.1 & 8 & 9.2 & $>0.05$ \\
\hline Asymmetry & 2 & 2.3 & 1 & 1.1 & $>0.05$ \\
\hline Deviation & 12 & 13.8 & 1 & 1.1 & $>0.05$ \\
\hline Others ${ }^{*}$ & 19 & 21.8 & 8 & 9.2 & $>0.05$ \\
\hline
\end{tabular}

*Other includes headaches and muscle tenderness

Table 1: Distribution according to TMJ signs and symptoms (87 joints) at pre-/ postoperative stages at 6 month follow up.

The mean average preoperative IID, right and left lateral excursions were $29.2 \mathrm{~mm}, 5.9 \mathrm{~mm}$, and $6.5 \mathrm{~mm}$ respectively. When compared with postoperative results, there was a significant improvement $(\mathrm{P}<0.05)$ in the IID $(34.4 \mathrm{~mm})$. Both right lateral excursion $(7.4 \mathrm{~mm})$ and left lateral excursion $(7.3 \mathrm{~mm})$ also showed improvement (Figure 1). A significant relationship between preoperative IID and MRI results was also found $(\mathrm{P}<0.05)$ as the IID in patients with ADDR presented with a median distance of $30 \mathrm{~mm}$ while the IID in patients with ADD showed a median distance of $26.5 \mathrm{~mm}$.
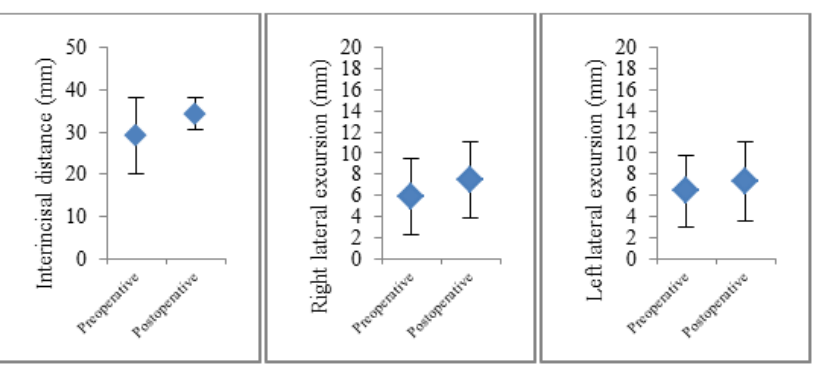

Figure 1: Mean value and standard deivation (SD) of pre- and postoperative interincisal distance and lateral excursion.

\section{MRI findings and clinical presentations}

Of the 87 cases, 37 (42.5\%) were diagnosed as ADDR, 45 (51.7\%) were diagnosed as ADD, 4 (4.6\%) had discs in normal position and 1 case had an unidentified disc position (Figure 2 (A and B)). When comparing preoperative results with MRI findings, $42.0 \%$ of cases with symptoms of pain had ADDR (as found by MRI) and $53.1 \%$ of cases with symptoms of pain had ADD. The difference between pain and disc position was not found to be statistically significant.

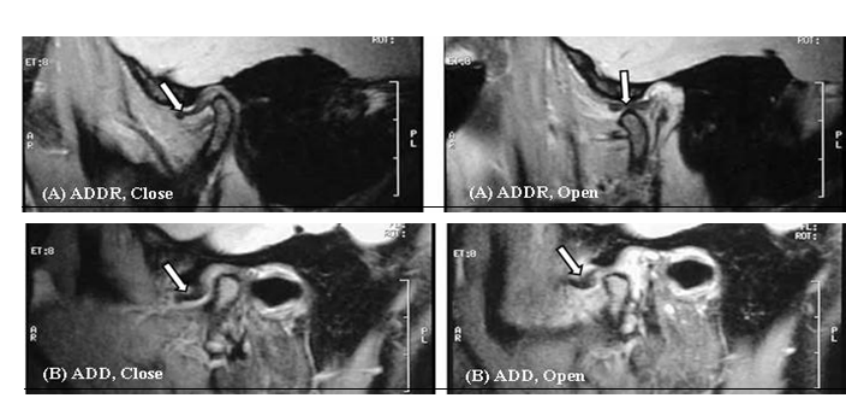

Figure 2: MRI photo showing anterior disc displacement (arrows) (A) with reduction and (B) without reduction.

Post arthroscopic results showed $40.5 \%$ of cases with symptoms of pain had ADDR and $44.4 \%$ of cases with symptoms of pain had ADD and $2(2.3 \%)$ of the cases with disc in the normal position also experienced postoperative pain. Based on statistical analysis, both preand post-operative pain had no meaningful association with the position of the disc as seen by MRI. 


\section{Arthroscopic, wilkes and clinical presentations}

Intraoperative arthroscopic findings are listed in Table 2. Arthroscopic findings showed adhesions (88.5\%), hyperemia (47.1\%), and synovitis $(31.0 \%)$ regardless of internal derangement stage. Three (3.4\%) cases exhibited disc perforations and fragmentations which corresponded with patients' symptoms of severe tenderness, grating, popping and dysfunction. These cases were later treated with open arthroplasty, discectomy and eminectomy. When comparing clinical symptoms with arthroscopic findings; pain, the chief preoperative complaint, was associated with adhesions, hyperemia and synovitis in $92 \%, 56 \%$ and $37 \%$ of cases respectively. Statistical analysis showed preoperative pain was positively correlated with the Wilkes Classification $(\mathrm{P}<0.05)$.

\begin{tabular}{|c|c|c|c|c|}
\hline \multirow{2}{*}{$\begin{array}{l}\text { Arthroscopic } \\
\text { finding }\end{array}$} & $\begin{array}{l}\text { Wilkes stage } \\
2\end{array}$ & $\begin{array}{l}\text { Wilkes stage } \\
3\end{array}$ & $\begin{array}{l}\text { Wilkes stage } \\
4\end{array}$ & $\begin{array}{l}\text { Unspecifi } \\
\text { ed }\end{array}$ \\
\hline & $(n=12)$ & $(n=18)$ & $(n=13)$ & $(n=44)$ \\
\hline Adhesions & 12 & 17 & 11 & 37 \\
\hline Hyperemia & 9 & 11 & 6 & 15 \\
\hline Synovitis & 4 & 4 & 10 & 9 \\
\hline Disc perforation & 0 & 0 & 0 & 3 \\
\hline Others ${ }^{*}$ & 0 & 1 & 3 & 11 \\
\hline
\end{tabular}

Table 2: Arthroscopic findings.

Based on the Wilkes stages in relation to clinical findings, a significant decrease in preoperative IID was observed as the Wilkes staging increased $(\mathrm{P}<0.05)$. Even though, postoperative results showed an improvement in IID in relation to the Wilkes staging (inverse correlation) (Figure 3), this relationship was not statistically significant.

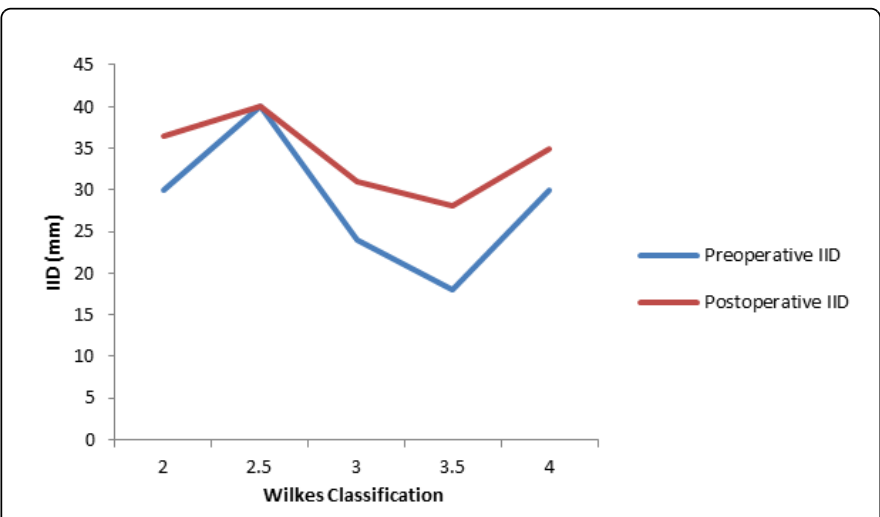

Figure 3: Median Inter incisal distance $(\mathrm{mm})$ pre- and postoperative.

Temporary post arthroscopy TMJ tenderness, facial nerve weakness, TMJ infection were seen in 13 (14.9\%), 3 (3.4\%) and $1(1.1 \%)$ of cases respectively. All complications resolved within the first week following arthroscopy and no indications of permanent injury were reported during the follow up period of this study.

\section{MRI and arthroscopic findings}

Magnetic resonance imaging diagnosed 37 (42.5\%) joints with ADDR and 45 (51.7\%) joints with ADD (Figure 4). The most common arthroscopic findings in patients with ADDR were adhesions, hyperemia and synovitis which were found in 23 (62.2\%), 13 (35.1\%) and $9(24.3 \%)$ of the joints respectively. In patients presenting with $\mathrm{ADD}$, cases of adhesions, hyperemia, and synovitis were 36 (80\%), 24 (53.3\%), and $17(37.8 \%)$ respectively. Other arthroscopic findings which were seen infrequently included disc perforation (3.4\%) and fragmentation. Although there appears to be a trend in higher percentages of adhesions, hyperemia and synovitis in cases of ADD as compared to ADDR, no significant correlation was found.

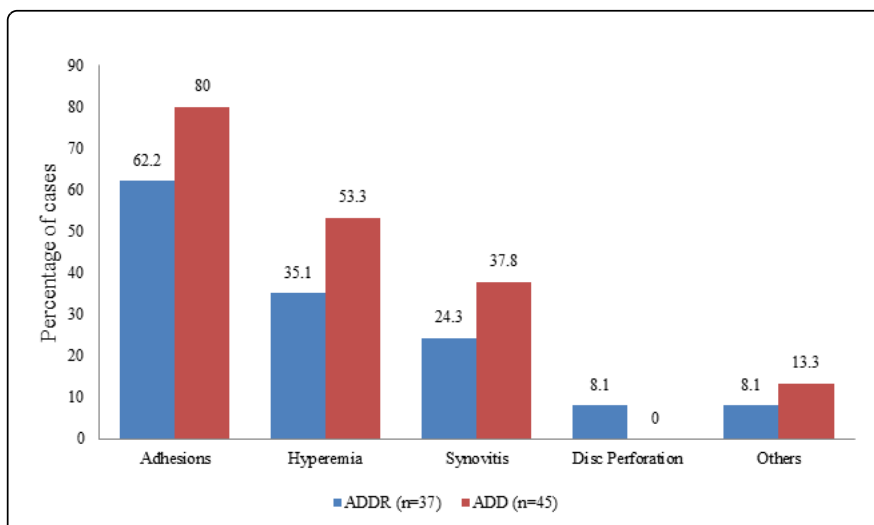

Figure 4: Percentage of adhesions, hyperemia, synovitis, disc perforations and others in patients with ADD and ADDR.

\section{Discussion}

Approximately 10 million individuals in the United States are affected by TMD with middle aged women representing $80 \%$ of patients being treated $[3,8]$. The average age and sex distribution of our study population was consistent with these previous studies.

In several studies, the measurement of successful treatment was based on two criteria: improvement of range of motion and reduction of pain levels [3-5,9] In regards to range of motion, our study found a significant $(\mathrm{P}<0.05)$ improvement in the IID; both lateral excursions showed improvement although not statistically significant. This correlates closely with studies by Ahmed et al. [3] and Smolka et al. [4] who found remarkable increase in mouth opening and reduction of pain in each Wilkes stages post arthroscopy. Further analysis of the preoperative data indicated an inverse relationship between preoperative IID and Wilkes Classification $(\mathrm{P}<0.05)$ as cases presenting with higher Wilkes stages correlated with lower IID on average. This conclusion is supported by Gonzalez-Garcia et al. [9] in their study comparing range of motion and Wilkes stages postoperatively at 1,3 and 6 months. They found a highly statistically significant difference $(\mathrm{P}<.001)$ among Wilkes stages with advanced stages showing progressively lower the IID values.

The results of this study showed conclusive evidence supporting the improvement of pain during our follow up period from $93.1 \%$ to $42.5 \%$ postoperatively $(\mathrm{P}<0.05)$. Other studies have shown results with the reduction of pain being statistically significant as well $[5,8,9]$ Leibur et al. [8] found that arthroscopic lysis and lavage for the treatment of TMD offered favourable long-term stable results for increased IID and 
Page 4 of 4

reduced pain. Both Gonzalez-Garcia et al. [9] and Murakami et al. [5] found significant decreases in TMJ pain after arthroscopy $(\mathrm{P}<.001)$ in long term studies with follow ups from 2 to 10 years $[5,9]$.

There was no correlation between pain and disc position as inferred by the MRI; position of the disc was not an indicator of the pain symptoms presented by patients both pre and post arthroscopy. This observation is consistent with those of other studies done by Emshoff et al. [2] In 2001, they compared the agreement between the presence of TMJ pain and the MRI diagnoses of ID, ADDR and ADD, They found poor agreement between the clinical and the MR imaging findings. Emshoff et al. concluded that the position of the disc on MRI does not correlate with treatment outcomes or symptoms of patients with TMJ pain. Unfortunately, cause of pain symptoms in patients with disc displacement is not fully understood. Although disc displacement plays a substantial role in the pain process, it is not always associated with pain. Several studies have shown that disc displacement can also occur in symptom-free joints [2]. Deviations in disc position alone is therefore not the only factor in the development of pain. Studies have shown that disc displacement, combined with dysfunction or inflammatory reactions in the TMJ [1,2] are key issues for pain symptoms.

This study revealed a significant relationship between IID of patients with ADD and ADDR preoperatively. Preoperative mean average interincisal distances of $34.0 \mathrm{~mm}$ and $25.6 \mathrm{~mm}$ were reported in the ADDR and ADD groups respectively. This agrees with a study by Montgomery et al. [10] which also found that, the ADD subjects had comparatively less preoperative ROM compared with the ADDR group. Although there was a significant improvement between pre and postoperative IID, no differences between postoperative interincisal distances of ADDR (34.2 mm) and ADD (34.1 mm) were found.

One confounding variable that must be considered in all studies is the presence or absence of stress-induced parafunctional habits. If parafunctional habits are not addressed or controlled, patients will likely suffer postoperative complications regardless of the outcome of the arthroscopy and treatment. We cannot conclude whether the pain and interincisal distances achieved postoperatively were partially the result of parafunctional habits. Further research is needed to determine the contributing factors of parafunctional habits on the successful outcomes of arthroscopy and TMD therapy.

Our investigation found arthroscopic lysis and lavage to significantly improve TMJ range of motion upon opening. The Wilkes Classification correlated with both preoperative IID and pain. No relationship was observed between MRI and arthroscopic results as all joints with disc displacement, with and without reduction, showed variable degrees of joint adhesion and hyperemia. This suggests that MRI should not be considered a necessity before TMJ arthroscopy. We agree that arthroscopy lysis and lavage in TMD patients continues to have an important role in the management of specific TMJ disorders however appropriate case selection is mandatory for successful surgical intervention to achieve the desired outcomes of pain relief and functional improvement.

\section{References}

1. Carrara SV, Conti PC, Barbosa JS (2010) Statement of the 1st consensus on temporomandibular disorders and orofacial pain. Dent Press J Orthod 15: 114-120.

2. Emshoff R, Innerhofer K, Rudisch A, Bertram S (2001) Relationship between temporomandibular joint pain and magnetic resonance imaging findings of internal derangement. Int J Ora Maxillofac Surg 30: 18-22.

3. Ahmed N, Sidebottom A, O'Connor M, Kerr HL (2012) Prospective outcome assessment of the therapeutic benefits of arthroscopy and arthrocentesis of the temporomandibular joint. Br J Oral Maxillofac Surg 50: 745-748.

4. Smolka W, Yanai C, Smolka K, Iizuka T (2008) Efficiency of arthroscopic lysis and lavage for internal derangement of the temporomandibular joint correlated with Wilkes classification. Oral Surg Oral Med Oral Pathol Oral Radiol Endod 106: 317-333.

5. Murakami K, Segami N, Okamoto M, Yamamura I, Takahashi K, et al. (2000) Outcome of arthroscopic surgery for internal derangement of the temporomandibular joint: long-term results covering 10 years. J Craniomaxillofac Surg 28: 264-271.

6. Ohnuki T, Fukuda M, Iino M, Takahashi T (2003) Magnetic resonance evaluation of the disk before and after arthroscopic surgery for temporomandibular joint disorders. Oral Surg Oral Med Oral Pathol Oral Radiol Endod 96: 141-148.

7. Wilkes $\mathrm{CH}$ (1989) Internal derangements of the temporomandibular joint: Pathological variations. Arch Otolaryngol Head Neck Surg 115: 469-477.

8. Leibur E, Jagur O, Müürsepp P, Veede L, Voog-Oras U (2010) Long-term evaluation of arthroscopic surgery with lysis and lavage of temporomandibular joint disorders. J Craniomaxillofac Surg 38: 615-620.

9. González-García R, Rodríguez-Campo FJ (2011) Arthroscopic Lysis and Lavage Versus Operative Arthroscopy in the Outcome of Temporomandibular Joint Internal Derangement: A Comparative Study Based on Wilkes Stages. J Oral Maxillofac Surg 69: 2513-2524.

10. Montgomery MT, Van Sickels JE, Harms SE (1991) Success of temporomandibular joint arthroscopy in disk displacement with and without reduction. Oral Surg Oral Med Oral Pathol 71: 651-659. 